Int. J. Morphol.,

36(3):1070-1074, 2018

\title{
Variaciones del Músculo Palmar Largo en la Población de Caldas (Colombia)
}

\author{
Variations of the Palmaris Longus Muscle in the Population of Caldas (Colombia)
}

\author{
Jorge Eduardo Duque Parra ${ }^{1,2}$; John Barco Ríos ${ }^{1}$ \& Diego Alejandro Gómez Castrillón ${ }^{1}$
}

DUQUE, P. J. E.; BARCO, R. J. \& GÓMEZ, C. D. A. Variaciones del músculo palmar largo en la población de Caldas (Colombia). Int. J. Morphol., 36(3):1070-1074, 2018.

RESUMEN: El músculo palmar largo es de los músculos más variables en su presentación en el cuerpo humano. El propósito del estudio fue determinar su grado de agenesia en una muestra de individuos del Departamento de Caldas (Colombia). Se evaluó la presencia del músculo palmar largo por visualización directa de su tendón, para lo cual se aplicó el test de Thompson, y cuando fue necesario se aplicó el Test de Schaeffer, en una muestra de 115 individuos de sexo femenino y masculino entre los 17 a 50 años de edad oriundos de Caldas. La agenesia global del palmar largo se encuentra en el 22,6 \% de la población caldense, pero la agenesia bilateral sólo se presenta en el 8,69 \% de los individuos, siendo mayor su presentación en las mujeres; mientras que la agenesia unilateral es más frecuente, por el orden del 13,91\%, guardando una proporción semejante entre hombres y mujeres. Aunque existe cierta concordancia con la mayoría de estudios realizados en otros países, nuestro estudio destaca dos hechos relevantes, que la agenesia global músculo del palmar largo tiene una de las frecuencias más elevadas de presentación, y que la agenesia unilateral, tanto derecha como izquierda, se encuentra entre los rangos más bajos. La agenesia global del músculo, bilateral y unilateral, es más común en las mujeres. Así mismo, la agenesia bilateral es más frecuente en las mujeres, con una presentación que duplica la de los hombres, mientras que la agenesia unilateral es proporcionalmente igual en ambos sexos.

PALABRAS CLAVE: Agenesia; Anatomía; Músculo; Músculo palmar largo.

\section{INTRODUCCIÓN}

El propósito de este estudio fue determinar el porcentaje de presentación del músculo palmar largo, el cual está localizado en la cara anterior del antebrazo (Alves et al., 2011; Gómez Castrillón, 2013). Se caracteriza por ser un músculo delgado, con un vientre corto e intercalado entre los músculos flexor radial del carpo y flexor ulnar del carpo (García-Porrero \& Hurlé, 2005), y se continúa con un tendón de longitud variable. Este músculo se origina en el epicóndilo medial del húmero (Testut \& Latarjet, 1967; García-Porrero \& Hurlé; Dauber, 2007; Moore et al., 2010), mientras que su inserción la realiza en la cara anterior y en el vértice de la aponeurosis palmar (Testut \& Latarjet; O’Rahilly, 1989; Rouvière \& Delmas, 2001; Dauber; Moore et al.). Su acción permite tensar la aponeurosis palmar al cerrar el puño (Testut \& Latarjet; García-Porrero \& Hurlé; Dauber; Moore et al.) y también ayuda a flexionar la palma de la mano (Testut \& Latarjet; Mbaka \& Ejiwunmi, 2009; Moore et al.), la muñeca (García-Porrero \& Hurlé) y el antebrazo.
La valoración del músculo palmar largo puede hacerse utilizando el test de Thompson, que consiste en empuñar la mano y flexionar la muñeca. También se puede emplear el test de Schaeffer, que consiste en hacer oposición entre el pulgar y el dedo mínimo dejando la muñeca parcialmente flexionada (Mbaka \& Ejiwunmi). En la literatura se reportan múltiples variantes anatómicas del músculo palmar largo, como las siguientes: puede presentarse en forma de tejido fibroso en toda su extensión o ser totalmente carnoso en toda su extensión (Testut \& Latarjet); puede ser tendinoso proximalmente y muscular distalmente, o presentarse doble (Testut \& Latarjet; Clemente, 1985); también puede estar conformado por dos vientres musculares conectados por un tendón en su parte intermedia, o presentar fascículos supernumerarios de músculos antebraquiales, o puede continuarse con la aponeurosis palmar sin fijarse al retináculo flexor (Testut \& Latarjet). Otra variante poco común es su presentación de manera invertida, es decir que su parte tendinosa está en la parte superior del antebrazo y su porción carnosa en la

\footnotetext{
${ }^{1}$ Universidad de Caldas, Programa de Medicina, Departamento de Ciencias Básicas, Manizales, Colombia.

${ }^{2}$ Universidad de Manizales, Programa de Medicina, Manizales, Colombia.
} 
parte inferior (Salgado et al., 2012; Twoon et al., 2017). Puede presentar distintos puntos de inserción, ya sea en las eminencias tenar e hipotenar, en el metacarpo, en los tendones de los músculos flexores de los dedos, en el tendón del flexor ulnar del carpo y en los huesos pisiforme o escafoides del carpo (Testut \& Latarjet) o en la aponeurosis palmar (Kumar et al., 2014). Una variante poco común es la presencia de un doble tendón (Iqbal et al., 2015).

Como se puede apreciar, el músculo palmar largo es de los músculos más variables del cuerpo humano (Clemente), y a menudo puede estar ausente (Testut \& Latarjet; Clemente; Rouvière \& Delmas; Moore et al.; Alves et al.) en el 10-14\% de las personas (Rouvière \& Delmas; GarcíaPorrero \& Hurlé), o estar presente en uno o en ambos miembros (normalmente en el lado izquierdo) (Moore et al.), hecho que se relaciona con el lado del cuerpo, el sexo y el grupo étnico ( $\mathrm{O}^{`}$ Rahilly). También puede encontrarse doble (dos vientres) o como músculo accesorio (Koo \& Roberts, 1997). La agenesia del músculo palmar largo es variable entre los diversos grupos étnicos, por lo que se debe tener en cuenta su prevalencia en la población que se esté estudiando (Sebastin et al., 2005a,b), así: $5 \%$ en chinos, $22 \%$ en japoneses, $2,7 \%$ en negros, $5 \%$ en blancos, $15 \%$ en ingleses y $18 \%$ en franceses (Testut \& Latarjet). En la población nigeriana, la agenesia de este músculo tiene marcada variación racial, en la que la prevalencia de la ausencia unilateral y bilateral es del 6,7 \% (Mbaka \& Ejiwunmi). En la población chilena la agenesia unilateral es de $11 \%$ y bilateral en un $9 \%$, siendo más frecuente en mujeres (Alves et al.).

Dos estudios realizados en Colombia muestran que, en Bucaramanga, la agenesia global es de $20,3 \%$, con un predominio de agenesia bilateral del 10,3\% (Ballesteros Acuña \& Saldariaga Tellez, 2002); mientras que una prueba piloto realizada en Caldas mostró una agenesia global del 25 \% (Gómez Castrillón). La mayor prevalencia de agenesia del palmar largo se registró en Turquía (Gaziantan) con $63,9 \%$, en tanto que la menor prevalencia se presentó en Corea con el 0,6 \% (Ceyhan \& Mavt, 1997). La ausencia del músculo palmar largo puede estar asociada con algunas morbilidades, como el síndrome del túnel carpiano, el síndrome del compartimiento de la muñeca y el síndrome del canal de Guyon (Saied \& Karamoozian, 2009; Salgado et al.).

Este músculo es útil en cirugía por su largo tendón, su diámetro, su fácil disponibilidad y porque al extraerlo no produce alteración funcional. Debido a estas características se le emplea en cirugía plástica reconstructiva, en cirugía de mano para injertos de tendón (Sebastin et al., 2005a,b; Twoon et al.), en aumento de labios (Davidson,
1995), en corrección de ptosis (Chauhan, 2003), en la parálisis facial (Atiyeh et al., 1998), en el síndrome del túnel carpiano grave (Park et al., 2010) y en la artroplastia de resección causada por la enfermedad de Kienbock (Küçük et al., 2011). Los anestesiólogos lo tienen en cuenta para realizar bloqueo nervioso de los nervios ulnar y mediano (Scott, 1995); además se emplea para la administración de fármacos, como corticoesteroides, para aliviar el dolor en el síndrome del túnel carpiano o en la artritis.

\section{MATERIAL Y MÉTODO}

Se realizó un estudio observacional cuantitativo para determinar el porcentaje de presencialidad del músculo palmar largo en una muestra de individuos del Departamento de Caldas (Colombia). Por conveniencia, se seleccionaron 115 individuos, 59 mujeres y 56 hombres, con edades comprendidas entre los 17 y 50 años. Como criterio de exclusión, no se seleccionaron aquellos individuos que hubieran sido tratados quirúrgicamente en alguna de sus regiones antebraquiales. Después de obtener su consentimiento informado, a cada individuo se le explicó el propósito del estudio y el procedimiento a desarrollar, que consistía en observar el relieve cutáneo que genera la parte muscular y tendinosa del músculo palmar largo, sin incidir el antebrazo. Se expusieron las dos regiones antebraquiales desde el codo hasta la muñeca y seguidamente se les implementó el test de Thompson, que consiste en empuñar la mano y flexionarla levemente con el pulgar sobre los demás dedos (Fig. 1), lo cual permite visualizar la presencia del tendón del músculo palmar largo por debajo de la piel. Cuando las circunstancias lo requirieron, se aplicó el test de Schaeffer, que consiste en oponer el pulgar y el dedo mínimo, colocando firme el antebrazo en flexión a 90 grados y con la muñeca parcialmente flexionada (Fig. 2), técnica que también permite visualizar el tendón del músculo. Los datos fueron consignados en una tabla previamente diseñada.

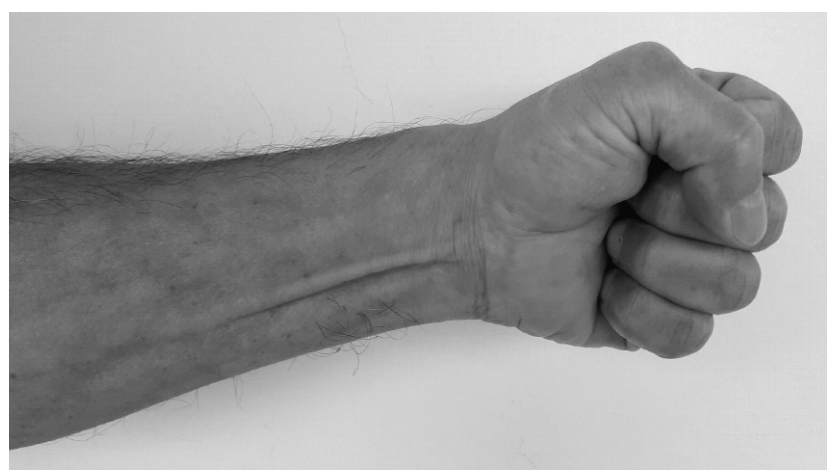

Fig. 1. Test de Thompson para observar la presencia del tendón del músculo palmar largo en el hombre. 


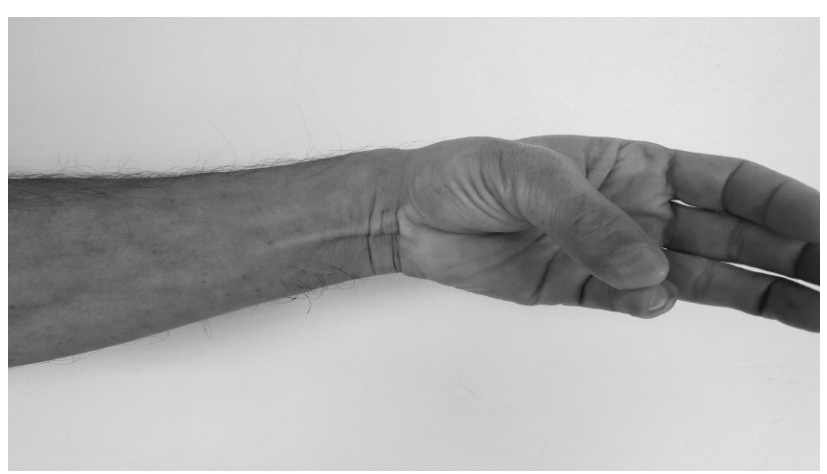

Fig. 2. Test de Schaeffer para observar la presencia del tendón del músculo palmar largo en el hombre.

\section{RESULTADOS}

De las 59 mujeres valoradas se encontró lo siguiente: en 44 de ellas $(74,57 \%)$ el músculo palmar largo estaba presente en ambos antebrazos, mientras que 7 (11,86 $\%)$ mostraron agenesia del músculo en los dos antebrazos. Otras $3(5,08 \%)$ tenían agenesia unilateral en el antebrazo izquierdo, y las 5 mujeres restantes $(8,47 \%)$ tenían agenesia unilateral en el antebrazo derecho.

Respecto a los 56 hombres valorados el resultado fue el siguiente: 45 de ellos $(80,35 \%)$ presentaron el músculo palmar largo en ambos antebrazos, mientras que 3 $(5,35 \%)$ presentaron agenesia bilateral. 5 hombres $(8,92$ $\%$ ) presentaron agenesia unilateral izquierda del palmar largo y los 3 restantes $(5,35 \%)$ presentaron agenesia unilateral derecha.

En general, se determinó que la presencia bilateral del músculo palmar largo en la población de Caldas es del $77,39 \%$, mientras que la agenesia global es de 22,6\%. Por otro lado, la agenesia bilateral del músculo es del 8,69 $\%$, en tanto que la agenesia unilateral izquierda corresponde al $6,95 \%$, mientras que la agenesia unilateral derecha es del 6,95\% de la población (Tabla I).

\section{DISCUSIÓN}

En las descripciones realizadas en los textos clásicos de anatomía sobre el músculo palmar largo, se informa que es un músculo muy variable e inconstante. Sin embargo, es importante aclarar el concepto de "inconstancia" para este músculo, pues da a entender que es muy común la agenesia del palmar largo en la población, lo que contrasta con lo reportado en múltiples trabajos de investigación realizados en distintos países y zonas geográficas, donde se demuestra que la agenesia se presenta en un porcentaje siempre inferior (Testut \& Latarjet; Mbaka \& Ejiwunmi; Alves et al.) comparado con aquella población que no tiene agenesia, salvo en una población turca (Gaziantan) donde la agenesia se presentó en un $63,9 \%$ de la población estudiada (Ceyhan \& Mavt).

Estudios completos sobre la inconstancia y variabilidad de presentación del citado músculo no se habían realizado antes en el Departamento de Caldas (Colombia). Los resultados de nuestro estudio reflejan que, en dicha región colombiana, el palmar largo tiene una frecuencia de presentación bilateral en el 77,39 \% de las personas analizadas, significativamente menor si lo comparamos con el promedio de los valores reportados en otros países asiáticos y europeos (Testut \& Latarjet; Mbaka \& Ejiwunmi; Alves et al.), que en promedio es del 87,45 \%; sin embargo, es un poco mayor comparado con el resultado obtenido por Morais et al. (2012) en la ciudad de Jataí (Brasil), donde la presencia bilateral es del $73,5 \%$. Lo anterior nos permite deducir que la agenesia del palmar largo en cualquiera de sus presentaciones, ya sea bilateral o unilateral, muestra un índice del 22,6 \%, lo que refleja una alta incidencia si lo comparamos con los valores reportados en otros países y con el realizado en Bucaramanga, Colombia (Ballesteros Acuña \& Saldariaga Tellez) quienes reportan un valor de 20,3\%. En cuanto a la agenesia bilateral del músculo en la población de Caldas, nuestro estudio registró un valor de 8,69\% en los individuos analizados, valor que está dentro de los rangos encontrados en otros países. Se observa además que la agenesia bilateral es más frecuente en las mujeres $(11,86 \%)$ que en los hombres $(5,35 \%)$, concordante con los estudios hechos en Bucaramanga (Ballesteros Acuña

Tabla I. Variaciones del músculo palmar largo en la población caldense de Colombia.

\begin{tabular}{lccccr}
\hline & Total analizados & Sin agenesia & Agenesia bilateral & Agenesia unilateral izquierda & Agenesia unilateral derecha \\
\hline Mujeres & 59 & $44(74,57 \%)$ & $7(11,86 \%)$ & $3(5,08 \%)$ & $5(8,47 \%)$ \\
Hombres & 56 & $45(80,35 \%)$ & $3(5,35 \%)$ & $5(8,92 \%)$ & $3(5,35 \%)$ \\
Totales & 115 & $89(77,39 \%)$ & $10(8,69 \%)$ & $8(6,95 \%)$ & $8(6,95 \%)$
\end{tabular}


\& Saldariaga Tellez) y en la India, que reportan un índice de agenesia mucho más elevado en las mujeres (Sankar et al., 2011).

Respecto a la agenesia unilateral, la encontramos en 16 individuos, que equivale al 13,91\% de la población estudiada, significativamente inferior a los valores reportados por otros autores en distintos estudios, como el de la India (70,5\%) (Sankar et al.) y el de Serbia $(57,6 \%)$ (Eric et al., 2010). En nuestro estudio, curiosamente hallamos una proporción semejante de agenesia unilateral, es decir 8 mujeres y 8 hombres, sólo que en las mujeres es un poco más marcada la agenesia en la parte derecha $(8,47 \%)$ que en la izquierda $(5,08 \%)$, mientras que en los hombres sucede lo contrario, es decir que es mayor en la parte izquierda $(8,92$ $\%)$ que en la derecha $(5,35 \%)$. Estos valores contrastan con lo reportado en otros estudios, donde se muestra que la agenesia unilateral es mucho más elevada en las mujeres (Eric et al.; Sankar et al.).

\section{CONCLUSIONES}

Nuestro estudio demuestra que, en el departamento de Caldas, la agenesia del músculo palmar largo en cualquiera de sus modalidades tiene el más alto índice de presentación, comparado con los demás países y poblaciones reportados en la literatura, excepto con la población de Gaziantan (Turquía). En Caldas, la agenesia global del músculo, es decir bilateral y unilateral, es más común en las mujeres. Así mismo, la agenesia bilateral es mucho más frecuente en las mujeres, con una presentación que duplica a la de los hombres, mientras que la agenesia unilateral es proporcionalmente igual en ambos sexos.

DUQUE, P. J. E.; BARCO, R. J. \& GÓMEZ, C. D. A. Variations of the palmaris longus muscle in the population of Caldas (Colombia). Int. J. Morphol., 36(3):1070-1074, 2018.

SUMMARY: The palmaris longus muscle is one of the most variable muscles in its presentation in the human body. The purpose of the study was to determine the degree of agenesis of this muscle in a sample of individuals in the Department of Caldas (Colombia). The presence of palmaris longus muscle by direct visualization of the tendon was evaluated by applying Thompson`s test and where necessary, Schaeffer`s test, in a sample of 115 individuals (female and male) between 17-50 years old, all natives of Caldas. The overall agenesis of palmaris longus muscle is in the $22.6 \%$, but bilateral agenesis occurs only in $8.69 \%$ of individuals, being higher in women, while unilateral agenesis is more common in the order of $13.91 \%$, keeping a similar proportion of men and women. Although there is some agreement with most of the studies made in other countries, our study highlights two important events, the global agenesis of palmaris longus muscle has one of the highest frequencies of submission, and that the unilateral agenesis, both right and left is among the lower ranks. Bilateral and unilateral global muscle agenesis is more common in women. Likewise, bilateral agenesis is more frequent in women, with a presentation that doubles that of men, while unilateral agenesis is proportionally equal in both sexes.

KEY WORDS: Agenesis; Anatomy; Palmaris longus muscle.

\section{REFERENCIAS BIBLIOGRÁFICAS}

Alves, N.; Ramírez, D.; Figueiredo, D. N. Study of frequency of the palmaris longus musclem in chilean subjects. Int. J. Morphol., 29(2):485-9, 2011. Atiyeh, B. A.; Hashim, H. A.; Hamdan, A. M.; Kayle, D. I. \& Moucharafieh, R. S. Lower reconstruction and restoration of oral competence with dynamic palmaris longus vascularized sling. Arch. Otolaryngol. Head Neck Surg., 124(12):1390-2, 1998.

Ballesteros Acuña, L. E. \& Saldariaga Tellez, V. Frecuencia del músculo palmar largo. Un estudio con población de Bucaramanga. Salud UIS, 34(1):12-7, 2002.

Ceyhan, O. \& Mavt, A. Distribution of agenesis of palmaris longus muscle in 12 to 18 years old age groups. Indian J. Med. Sci., 51(5):156-60, 1997.

Chauhan, R. Atypical innervation of palmaris longus - A case report. $J$. Anat. Soc. India, 52(2):171-3, 2003.

Clemente, C. D. Gray's Anatomy. 30a ed. Philadelphia, Lea \& Febiger, 1985. pp.531-2.

Dauber, W. Pocket Atlas of Human Anatomy. Based on the International Nomenclature. $5^{\text {a }}$ ed. New York, Thieme, 2007. pp.114.

Davidson, B. A. Lip augmentation using the palmaris longus tendon. Plast. Reconstr. Surg., 95(6):1108-10, 1995.

Eric, M.; Krivokuca, D.; Savovic, S.; Leksan, I. \& Vucinic, N. Prevalence of the palmaris longus through clinical evaluation. Surg. Radiol. Anat., 32(4):357-61, 2010.

García-Porrero, J. A. \& Hurlé, J. M. Anatomía Humana. Madrid, McGrawHill Interamericana, 2005. pp.196.

Gómez Castrillón, D. A. Variaciones del músculo palmar largo en la población colombiana. Rev. Argent. Anat. Online, 4(Supl. 4):64, 2013.

Iqbal, S.; Iqbal, R. \& Iqbal, F. A bitendinous palmaris longus: Aberrant insertions and its clinical impact - A case report. J. Clin. Diagn. Res., 9(5):AD03-5, 2015.

Koo, C. C. \& Roberts, A. H. The palmaris longus tendon. Another variation in its anatomy. J. Hand Surg. Br., 22(1):138-9, 1997.

Küçük, L.; Ozdemir, O.; Coskunol, E.; Sügün, T. S. \& Ozaksar, K. The effect of excisional arthroplasty with palmaris longus tendon on carpal height ratio in Stage 3 Kienböck's disease. Acta Orthop. Traumatol. Turc., 45(6):393-8, 2011.

Kumar, N.; Patil, J.; Swamy, R. S.; Shetty, S. D.; Abhinitha, P.; Rao, M. K. \& Nayak, S. B. Presence of multiple tendinous insertions of palmaris longus: a unique variation of a retrogressive muscle. Ethiop. J. Health Sci., 24(2):175-8, 2014.

Mbaka, G. O. \& Ejiwunmi, A. B. Prevalence of palmaris longus absence a study in the Yoruba population. Ulster Med. J., 78(2):90-3, 2009.

Moore, K. L.; Dalley, A. F. \& Agur, A. M. R. Anatomía con Orientación Clínica. $6^{\mathrm{a}}$ ed. Baltimore, Lippincott Williams \& Wilkins, 2010. pp.749.

Morais, M. A.; Gomes, M. S.; Helrigle, C.; Malysz, T. Prevalence of agenesis of the palmaris longus muscle in Brazil and its clinics correlation. $J$. Morphol. Sci., 29(4):238-42, 2012.

O`Rahilly, R. Anatomía. 5ª ed. Ciudad de México, McGraw-Hill, 1989. pp. 142 . 
Park, I. J.; Kim, H. M.; Lee, S. U.; Lee, J. Y. \& Jeong, C. Opponensplasty using palmaris longus tendon and flexor retinaculum pulley in patients with severe carpal tunnel syndrome. Arch. Orthop. Trauma Surg., 130(7):829-34, 2010.

Rouvière, H. \& Delmas, A. Anatomía Humana, Descriptiva, Topográfica y Funcional. Tomo III: Miembros. Sistema nervioso Central. $10^{\mathrm{a}} \mathrm{ed}$. Paris, Masson, 2001. pp.109.

Saied, A. \& Karamoozian, S. The relationship of presence or absence of palmaris longus and fifth flexor digitorum superficialis with carpal tunnel syndrome. Eur. J. Neurol., 16(5):619-23, 2009.

Salgado, G.; Cantín, M.; Inzunza, O.; Muñoz, A.; Saez, J. \& Macuer, M. Bilateral reversed palmaris longus muscle: a rare anatomical variation. Folia Morphol. (Warsz), 71(1):52-5, 2012.

Sankar, K. D.; Bhanu, P. S. \& John, S. P. Incidence of agenesis of palmaris longus in the Andhra population of India. Indian J. Plast. Surg., 44(1):134-8, 2011.

Scott, S. B. Techniques of Regional Anaesteshia. Connecticut, Appleton \& Lange/Mediglobe, 1995. pp.112-3.

Sebastian, S. J.; Lim, A. Y. T.; Bee, W. H.; Wong, T. C. M. \& Methil, B. V. Does the absence of the palmaris longus affect grip and pinch strength? J. Hand Surg. Br., 30(4):406-8, 2005b.

Sebastin, S. J.; Puhaindran, M. E.; Lim, A. Y.; Lim, I. J. \& Bee, W. H. The prevalence of absence of the palmaris longus--a study in a Chinese population and a review of the literature. J. Hand Surg. Br., 30(5):5257, 2005a.

Testut, L. \& Latarjet, A. Tratado de Anatomía Humana. Barcelona, Salvat, 1967. pp.1029-30.

Twoon, M.; Jones, C. D.; Foley, J. \& Davidson, D. Reversed palmaris longus muscle: a report of two cases. Case Reports Plast. Surg. Hand Surg., 4(1):73-6, 2017.

\author{
Dirección para correspondencia: \\ Jorge Eduardo Duque Parra \\ Departamento de Ciencias Básicas \\ Programa de Medicina \\ Universidad de Caldas \\ Sede principal \\ Calle 65 No. 26-10 \\ Manizales \\ Colombia
}

Email: jduqueparra@yahoo.com.mx

Recibido : 24-02-2018

Aceptado: 09-06-2018 